\section{Regards sur l'économie allemande}

Bulletin économique du CIRAC

$72 \mid 2005$

Varia

\title{
La cogestion allemande à l'épreuve de la globalisation
}

René Lasserre

\section{(2) OpenEdition}

\section{Journals}

Édition électronique

URL : http://journals.openedition.org/rea/246

DOI : $10.4000 /$ rea. 246

ISBN : 978-2-8218-0840-9

ISSN : 1965-0787

\section{Éditeur}

CIRAC

\section{Édition imprimée}

Date de publication : 1 juillet 2005

Pagination : 7-16

ISSN : 1156-8992

\section{Référence électronique}

René Lasserre, "La cogestion allemande à l'épreuve de la globalisation », Regards sur l'économie allemande [En ligne], 72 I juillet 2005, document 1, mis en ligne le 22 avril 2008, consulté le 19 avril 2019. URL : http://journals.openedition.org/rea/246 ; DOI : 10.4000/rea.246 


\title{
La cogestion allemande à l'épreuve de la globalisation
}

\author{
René Lasserre
}

\begin{abstract}
En Allemagne, le modèle social a cessé d'être une "valeur-refuge " et se trouve décidément soumis à rude inventaire. La cogestion qui constitue en Europe le système le plus avancé de participation des salariés aux décisions de l'entreprise et qui est l'un des éléments les plus spécifiques du système allemand de relations professionnelles fait à son tour l'objet d'une sévère remise en question. C'est en particulier la représentation des salariés à parité de sièges et avec voix délibérative dans les conseils de surveillance des grandes sociétés qui est aujourd'hui sur la sellette.
\end{abstract}

Depuis un certain temps déjà, les chefs d'entreprise et les milieux d'affaires dénoncent le caractère contraignant d'un dispositif qui complique et ralentit les choix stratégiques de l'entreprise et handicape sa réactivité dans la compétition globale. Mais au cours de la période récente, les critiques n'ont cessé de s'amplifier pour dénoncer l'anachronisme croissant de cette "exception allemande " face aux exigences nouvelles qu'imposent la libre circulation des capitaux et l'intégration européenne. La présence de représentants salariés mandatés et a fortiori de syndicalistes de premier plan dans les organes de contrôle des sociétés défie les règles d'une gouvernance soucieuse de rentabilité et de création de valeur. Après quelques autres, la crise qui secoue actuellement la direction de Volkswagen vient illustrer les ambivalences d'un système qui, sous couvert de démocratie, ne garantit ni transparence, ni efficacité. Par ailleurs, l'avancée récente du droit européen des sociétés, avec l'adoption du statut de la société de droit européen et sa transposition en droit allemand, fait apparaitre l'isolement croissant dans lequel la cogestion place les groupes allemands dans un processus de fusions-acquisitions appelé à s'amplifier. Autant d'enjeux très controversés du débat sur la compétitivité qui appellent à repenser un système de gouvernance conçu dans les années 1960 et qui, à la veille d'une échéance électorale décisive, mettent désormais sa réforme à l'ordre du jour.

A côté d'un système de négociation collective fortement structuré fondé sur l'autonoie contractuelle des organisations syndicales et patronales, la cogestion constitue le second pilier sur lequel repose le partenariat social allemand. Tandis que le dispositif contractuel assure aux organisations syndicales un réel pouvoir de négociation dans la fixation des salaires et des conditions de travail au niveau de la branche professionnelle, la cogestion garantit aux salariés la possibilité de faire valoir leurs intérêts dans l'entreprise en reconnaissant à leurs représentants un certain nombre de pouvoirs de co-décision.

Chacun des deux piliers présente de fortes particularités qui concourent à la singularité du système allemand de relations professionnelles, mais à des derés différents. L'importance de la régulation négociée fondée sur le contrat n'est certes pas une caractéristique spécifique à l'Allemagne, mais la cohérence de son système contractuel tient en grande partie à l'unité et à la représentativité des organisations professionnelles ainsi qu'à leur volonté partagée d'exercer pleinement les droits et responsabilités qui découlent de la liberté de coalition. Sur cette base s'est construit un équilibre institutionnel durable qui assure en

Cogestion : le second pilier du partenariat social allemand 
Egalité des droits entre capital et travail

Un modèle de gouvernance unique en Europe...

... mais aujourd'hui obsolète? pratique une parité de pouvoirs entre partenaires sociaux dans la régulation sociale.

Le système de cogestion répond lui aussi à une logique d'équilibre, mais découle d'une philosophie sociale spécifique, fondée sur le principe de l'égalité (Gleichberechtigung) des droits du capital et du travail et de la vocation conjointe des deux facteurs à co-déterminer (mitbestimmen) la marche de l'entreprise. La Mitbestimmung procède ainsi d'une construction historique et juridique originale qui se traduit par l'existence de régimes légaux de représentation qui, sous différentes formes et à des degrés variables selon les domaines, permettent aux salariés, par la voie de représentants élus ou désignés, d'exercer un droit de contrôle et de participer aux décisions relatives à l'organisation et à la gestion de l'entreprise. En cela, il instaure un partage du pouvoir entrepreneurial qui n'a pas d'équivalent en Europe et dans les sociétés industrielles développées et peut véritablement être considéré, en termes de respect des droits des travailleurs, comme l'un des éléments les plus originaux du modèle social allemand.

La cogestion allemande a longtemps fait figure de référence en Europe. Elle constituait une forme avancée de démocratie sociale avec la reconnaissance et la garantie des droits individuels et collectifs des salariés sur le lieu de travail. Elle apparaissait également comme un modèle de gouvernance et de management en ce qu'elle garantissait une concertation sociale permanente dans l'entreprise, facteur de cohésion et d'efficacité. Bien que novateur et précurseur, ce système, qui a été mis en place dans l'après-guerre mais dont le développement n'est arrivé à pleine maturité qu'à la fin des années 1970, n'a cependant pas réussi à faire école en Europe. Moins d'ailleurs parce qu'il a d'emblée fait figure d'anti-modèle dans les pays de culture entrepreneuriale et sociale différente, comme la France ou la Grande-Bretagne, que parce qu'au cours des deux dernières décennies, il n'a pas entièrement réussi à faire les preuves de son efficacité et de sa supériorité dans un environnement économique et social en pleine mutation.

De sorte qu'aujourd'hui, à l'heure de l'économie globale, la cogestion apparaît à bien des égards comme le vestige d'une 'exception sociale allemande' héritée d'une histoire sociale et politique particulière, comme une réponse datée, et en partie déphasée, face aux nouvelles exigences de la gouvernance d'entreprise au plan européen et international. La cogestion fait l'objet de sévères critiques et compte un nombre croissant de détracteurs qui voient en elle un handicap de compétitivité pour l'économie allemande, à la fois en termes de réactivité managérielle des entreprises et d'attractivité pour les investisseurs. Le diagnostic mérite cependant d'être nuancé selon qu'on considère les différents volets du système et qu'on se place sous l'angle du management stratégique, de la corporate governance ou de la gestion des ressources humaines. Sur ces trois registres, et à l'instar d'autres éléments constitutifs du modèle social allemand, la cogestion est néanmoins confrontée à des révisions douloureuses et à un avenir incertain.

\section{Un dispositif complexe et hétérogène}

Un dispositif à 2 niveaux

Le système allemand de cogestion est un édifice institutionnel à plusieurs étages qui est le fruit d'une gestation politique difficile et d'une stratification juridique progressive. La cogestion s'exerce à deux niveaux principaux, très nettement distincts par leurs domaines et leur champs d'application : celui de l'établissement (Betrieb) où s'organisent la production et la vie de travail, celui de l'entreprise (Unternehmen) en tant qu'entité juridique où se prennent les décisions stratégiques de gestion.

Etablissement : « cogestion simple"
Dans le premier cas, la participation des salariés est codifiée par un régime légal désigné sous le terme significatif de Constitution d'établissement (Betriebs- 
verfassung) qui définit les droits, devoirs et pouvoirs respectifs du conseil d'établissement, le Betriebsrat (BR) élu par le personnel, et de l'employeur. Ce régime, instauré en 1952 et plusieurs fois aménagé depuis, est un régime de droit commun s'appliquant à tous les établissements d'au moins 5 salariés ; il organise les rapports sociaux au quotidien, ce qui lui vaut l'appellation courante mais contestable de "petite cogestion » ou de "cogestion simple ». II reconnaît au BR des attributions très larges qui couvrent presque tous les aspects de la vie de l'établissement mais sont de portée très variable selon les domaines, allant d'un simple droit d'information jusqu'au droit de co-décision. C'est dans le domaine social que les pouvoirs du BR sont les plus conséquents : dans la plupart des questions touchant à la gestion collective du personnel et aux conditions de travail, son pouvoir de co-décision implique que l'employeur ne peut prendre de décision sans l'avoir préalablement consulté et obtenu son accord formel. Dans les questions de personnel touchant au contrat de travail individuel (embauche ou licenciement), le BR dispose d'un droit de contrôle suspensif sur la régularité et l'opportunité sociale de la mesure, lequel ouvre au salarié le droit de recours devant le juge (voir REA 62/03). Dans les questions économiques touchant à l'organisation de l'établissement ou de la production, le BR dispose d'un large pouvoir consultatif lui permettant d'émettre des avis et des propositions qui sont laissés à la libre appréciation de l'employeur. Mais dès qu'une décision économique implique des conséquences sociales tangibles pour les personnels, l'employeur est tenu de présenter un plan social d'accompagnement dont les mesures compensatoires doivent recueillir l'accord du Betriebsrat.

On le voit : il s'agit là d'un dispositif de concertation très élaboré qui instaure un pouvoir très largement partagé en matière de gestion, mais où la répartition des prérogatives respectives de l'employeur et des représentants du personnel est très finement équilibrée : l'autonomie de décision économique de l'employeur et ses prérogatives en matière de contrat de travail sont préservées, tandis que le Betriebsrat se voit investi d'un pouvoir d'information et de contrôle dans tous les domaines ou cas de figure où les décisions du chef d'entreprise affectent les droits et les intérêts sociaux des salariés. Ce système est l'un des plus élaborés d'Europe et ménage dans l'entreprise allemande un espace de dialogue extrêmement large sur la base duquel s'est développée, à grande échelle et dans la plupart des grands secteurs de l'économie, une gestion concertée des ressources humaines qui touche la quasi-totalité des grandes entreprises et environ trois quarts des PME dès lors qu'elles atteignent le seuil de 50 salariés.

Le second niveau d'exercice de la cogestion concerne les entreprises prises cette fois en tant que personnes morales. Le droit de cogestion des salariés s'exerce par l'intermédiaire de représentants élus ou désignés qui siègent avec voix délibérative aux côtés des représentants des propriétaires ou des actionnaires dans les instances de direction et de contrôle des sociétés. Réservée aux sociétés de taille plus importante, cette « grande cogestion » fait l'objet de trois régimes différents. Le plus ancien, instauré par une loi de 1951, concerne les entreprises sidérurgiques et minières de plus de 1000 salariés. II prévoit une représentation paritaire des salariés dans un conseil de surveillance présidé par un homme neutre et la présence dans le directoire d'un directeur du personnel nommé avec l'accord de la majorité des représentants des salariés du conseil de surveillance. Ce régime maximaliste, hérité de la période initiale de reconstruction, ne concerne plus aujourd'hui qu'une quarantaine de sociétés. A l'inverse, le régime instauré en 1952 dans le cadre du Betriebsverfassungsgesetz reste aujourd'hui très répandu puisqu'il concerne les entreprises de 500 à 2000 salariés, soit environ 3500 sociétés. Mais c'est en même temps un régime minimal, puisque la représentation salariée se limite au seul conseil de surveillance avec seulement un tiers des sièges.
Gestion concertée des ressources humaines

Entreprise comme personne morale : représentation salariale au conseil de surveillance 
Entreprises de plus de 2000 salariés : prééminence du capital au conseil de surveillance...

... mais possibilités d'influence considérables pour les salariés

Deux niveaux de légitimité
Enfin, le troisième dispositif instauré par la loi sur la cogestion de 1976 concerne essentiellement les grands groupes, puisqu'il s'applique à toutes les sociétés de plus de 2000 salariés, soit 746 entreprises employant au total un peu plus de 5 millions de salariés. II prévoit une représentation numériquement paritaire des salariés dans le conseil de surveillance ; elle est cependant tempérée par la présence d'un cadre dirigeant sur le banc des salariés et surtout par le fait que le président du conseil de surveillance est obligatoirement élu parmi les représentants des actionnaires et dispose d'une seconde voix décisive qui, en cas de partage égal des voix, assure la prééminence du capital.

S'il apparaît que les deux régimes de droit commun n'assurent pas véritablement la parité des pouvoirs du capital et du travail dans les conseils de surveillance des entreprises, il n'en demeure pas moins qu'ils ménagent aux représentants des salariés des possibilités d'influence considérables. Siégeant avec voix délibérative, à égalité de droits avec ceux des actionnaires, les représentants salariés ont accès à toutes les informations concernant la situation économique et financière de l'entreprise et sont associés à toutes les grandes décisions, que ce soit en matière de répartition des bénéfices, de politique d'investissement, de choix des dirigeants, d'évolution de l'actionnariat, d'opérations de fusion-acquisition et de projets nouveaux de développement. Même s'ils ne disposent pas d'un pouvoir suffisant pour infléchir la politique du groupe, ils ont la possibilité d'y faire valoir et prendre en compte les intérêts des salariés. Sans réellement permettre d'exercer un réel contre-pouvoir, la «grande cogestion » assure pour le moins une concertation sur les choix stratégiques de l'entreprise. Celle-ci constitue un atout précieux pour l'action des Betriebsräte qui pourront ensuite peser efficacement sur leur mise en œuvre et faire valoir les intérêts des salariés sur le terrain. Et ce d'autant plus qu'en règle générale, dans les grandes sociétés, le président du Betriebsrat siège également sur le banc des salariés au sein du conseil de surveillance.

Les deux dispositifs fonctionnent ainsi à des niveaux distincts et concernent des enjeux différents, bien que complémentaires. Ils présentent néanmoins une caractéristique commune qui est d'être l'un et l'autre fondé sur une représentation médiatisée des salariés. Que ce soit sur le lieu de travail ou dans les organes de contrôle de l'entreprise, la cogestion reste l'apanage de représentants élus ou désignés, agissant au nom des salariés Au niveau de l'établissement, la codécision s'exerce par l'intermédiaire exclusive du Betriebsrat dans le cadre d'un dialogue formalisé avec le responsable du personnel ou le directeur; elle intervient néanmoins en proximité relativement immédiate des salariés du fait du contact étroit que les élus entretiennent généralement avec le personnel et de l'impact tangible de leur action sur les conditions de travail et la vie sociale de l'établissement. De ce fait, la co-décision bénéficie généralement d'un enracinement solide et d'une légitimité très largement partagée sur le lieu de travail. C'est beaucoup moins vrai de la cogestion d'entreprise qui fait appel à des processus complexes de désignation des représentants élus des salariés au conseil de surveillance, un certain nombre étant extérieurs à l'entreprise et proposés par le syndicat. II en résulte une moindre proximité sinon un éloignement des élus dont le mandat s'exerce au niveau des états-majors et dont les retombées concrètes de l'action, en dehors des situations de crise, ne sont pas réellement ou immédiatement perceptibles par les personnels.

\section{La cogestion d'entreprise : un système en mal de crédibilité sociale}

Adoptée en 1976 puis instaurée en 1978 au terme d'un débat politique lancé 10 ans plus tôt à l'initiative du DGB et des grands syndicats d'industrie, la cogestion dans les grandes entreprises s'est établie sans difficulté ni tension majeure dans tous les grands secteurs de l'industrie et des services, reléguant progressivement le modèle charbon-acier au rang de système résiduel. Ce système n'a 
pas pour autant entièrement tenu ses promesses : alors que depuis les années 1960 le mouvement syndical l'avait inlassablement présenté comme la pierre angulaire de la démocratie économique et sociale, sa contribution à faire évoluer de façon tangible les rapports sociaux dans l'entreprise n'a pas été manifeste. II n'en avait d'ailleurs plus la vocation dans sa conception même. Alors que, dans les années 1980, les salariés aspiraient à participer directement à l'organisation de leur activité et de leur environnement de travail et que, parallèlement, les entreprises cherchaient à promouvoir leur initiative individuelle et leur capacité d'innovation par différentes formes de management participatif (cercles de qualité, etc.), la cogestion paritaire relevait plutôt d'une régulation collective centralisée, exercée par un co-pilotage institutionnalisé au sommet de l'entreprise. En cela, elle restait encore inspirée de la philosophie constitutionnaliste des années 1950 et se situait déjà en décalage patent face aux nouvelles aspirations des salariés et à l'émergence de formes nouvelles de management décentralisé.

Le contexte économique dans lequel elle a été introduite ne la rendait pas pour autant obsolète mais en a très largement déplacé les enjeux et les contenus, à l'instar de ce qui s'était produit dans le secteur charbon-acier, à partir des années 1960 où, après avoir efficacement servi la reconstruction de l'industrie lourde, la cogestion en a facilité la reconversion. Dans le contexte de compétition internationale accrue qui a succédé aux chocs pétroliers, elle a surtout permis de gérer en co-responsabilité, c'est-à-dire dans une logique socialement optimale, la rationalisation et la modernisation de l'outil de production des grands groupes de l'industrie et des services. Au cours des années 1980, la cogestion a ainsi fait ses preuves sur le terrain économique : avec le soutien des syndicats des principaux secteurs, les grands groupes industriels ont modernisé leurs structures de production et poursuivi une stratégie active d'internationalisation, les excédents records obtenus à l'exportation consolidant en retour l'emploi industriel en Allemagne.

Avec les charges de l'unification, la récession des années 1990 et la globalisation accélérée des échanges, la compétitivité allemande s'est par la suite fortement détériorée, et les positions des entreprises sur le marché européen et mondial ont été fortement secouées, tant par leurs concurrents européens et anglo-saxons que par les compétiteurs des nouveaux pays industriels. Dans ce nouveau contexte, la cogestion est devenue un instrument de gestion des restructurations qui a démontré son efficacité managérielle en imposant des réorganisations dont les effets en termes de conditions de travail et d'emploi ont été sévères. Loin de faire obstacle aux changements structurels, les représentations salariées dans les conseils de surveillance ont agi en pleine coresponsabilité et généralement soutenu les directions dans leurs projets de modernisation, que ce soit dans l'allègement et la décentralisation des structures de pilotage ou la réorganisation en profondeur des processus de production (externalisation, sous-traitance, flux tendus...). Au nom d'une productivité accrue et de la sauvegarde à long terme des emplois, les représentants salariés ont généralement accepté les réductions d'effectifs et les nouvelles formes d'organisation du travail qui en découlaient. La cogestion a certes rendu la prise de décision plus difficile en obligeant les directions des groupes à discuter et justifier leur choix stratégiques. Mais elle n'a généralement pas bloqué le processus, et dès lors que les dimensions sociales ont été raisonnablement prises en compte, la cogestion a plutôt contribué à justifier le bien-fondé des projets de réorganisation, à en faire partager les objectifs et à en faciliter la mise en œuvre.

Confrontée ensuite à la libéralisation du marché des capitaux et à la multiplication des fusions-acquisitions, la cogestion a dû faire face à des opérations de regroupement plus ou moins obligés, sans pour autant constituer, comme l'a montré le cas de figure de l'OPA Vodafone/Mannesman en 2000, un réel facteur de blocage et encore moins le rempart si souvent dénoncé à des
La cogestion a facilité les restructurations des années $1980 .$. .

... la réorganisation des process de production dans les années 1990...

... et les récents arbitrages en termes de temps de travail 
Un frein à la réactivité de l'entreprise

Incompatibilité avec la corporate governance opérations en capital, y compris d'investisseurs étrangers. Plus récemment encore, sur l'épineuse question des délocalisations, la cogestion s'est trouvée soumise à des arbitrages difficiles et conflictuels, mais a finalement permis, moyennant des sacrifices en termes de temps de travail et de revenu, de faire prévaloir des stratégies de préservation de l'emploi domestique. II n'en demeure pas moins que, si la cogestion au sein du conseil de surveillance offre encore à l'entreprise la possibilité d'apporter des réponses concertées aux mutations rapides induites par la globalisation des marchés, elle apparaît de plus en plus sous une dimension défensive et régressive, perdant ainsi en crédibilité et en légitimité auprès des salariés.

\section{Un modèle de gouvernance anachronique ?}

Face à l'ampleur des défis, le patronat allemand est conduit à son tour à réévaluer les avantages de la cogestion en terme d'efficacité managérielle. La concertation sur les choix stratégiques apparaît, dans la période difficile que traverse l'économie allemande et dans un contexte de compétition internationale extrêmement vive, comme une contrainte supplémentaire jugée de moins en moins supportable. Au point que certains de ses représentants les plus en pointe en revendiquent la suppression pure et simple, et que les deux organisations principales BDI et BDA, dans un rapport commun publié en novembre 2004, intitulé «Moderniser la cogestion», en préconisent une reconfiguration substantielle.

La critique principale vient du patronat industriel qui reproche principalement à la cogestion salariée dans le conseil de surveillance de compliquer et de retarder inutilement la prise de décision stratégique. Alors que l'entreprise est confrontée à un processus d'adaptation permanent qui implique des choix difficiles à opérer dans des délais de plus en plus courts, la cogestion constitue un frein à la réactivité de l'entreprise et un handicap à sa compétitivité. La cogestion allemande, conçue à une époque où les choix industriels s'inscrivaient dans la durée, met en jeu des processus de décision qui ne sont plus adaptés à une économie où le cycle de vie des produits se raccourcit, où les avantages compétitifs de l'entreprise se mesurent simultanément en terme d'innovation et en termes de coûts et exigent une adaptation rapide du processus de production. Dans ce contexte de mutation permanente, l'exigence de la prise en compte des intérêts sociaux des salariés qui, dans le système actuel, fonde la participation décisionnelle de ces derniers dans les choix économiques de l'entreprise, devrait faire appel à d'autres mécanismes, moins contraignants et plus flexibles. Au lieu d'une obligation formelle uniforme, la participation des salariés aux décisions stratégiques pourrait être laissée à l'initiative contractuelle des partenaires sociaux de l'entreprise. II devrait pouvoir relever d'un accord qui en fixerait les contenus et les modalités, tout en ménageant d'autres options, telles que le Conseil consultatif distinct que le modèle unitaire de gouvernance britannique prévoit à côté du Board of Directors.

L'offensive patronale met également en avant l'exotisme que constitue la cogestion allemande face à la nouvelle donne mondiale sur le marché des capitaux et à la culture de la shareholder value. Le régime de la cogestion de 1976 présente des incompatibilités flagrantes avec les règles et pratiques internationales de corporate governance qu'impose le marché des capitaux pour garantir la rentabilité et l'attractivité financières des grandes entreprises. En particulier, la présence dans les conseils de surveillance avec voix décisionnelle de membres salariés qui, à l'instar du président du Betriebsrat, exercent des fonctions de représentation unilatérale des intérêts des salariés au sein même de l'entreprise, est contraire aux principes d'indépendance et de transparence qui doivent désormais prévaloir dans les organes de contrôle des sociétés cotées. Quant à la présence de dirigeants syndicaux de tout premier plan dans le conseil de la plupart des sociétés allemandes cotées au DAX-30, elle fait tout 
simplement figure d'épouvantail dans le paysage financier international. La lourdeur et l'hétérogénéité des conseils de surveillance des sociétés cogérées, la complexité de leur fonctionnement apparaissent également comme un défi aux standards d'un contrôle opérationnel et efficace. L'absence quasi-totale d'administrateurs salariés étrangers dans les sociétés allemandes soumises à cogestion constitue une autre singularité tout à fait surprenante au regard du fait que, parmi elles, un tiers est sous contrôle de firmes étrangères et qu'une large majorité dispose d'implantations importantes en dehors d'Allemagne.

Ces constats plaident pour un aggiornamento rapide de la réglementation qui, sans aller jusqu'à une normalisation pure et simple du droit allemand des sociétés et à l'abandon du principe de cogestion, en accroisse très sensiblement la lisibilité et l'attractivité internationales. II s'agit avant tout d'éviter que, dans le nouveau contexte de libre circulation des capitaux et de concurrence entre les sites nationaux de production, la cogestion contribue à dissuader les entreprises étrangères de venir s'implanter en Allemagne.

\section{La cogestion allemande en Europe : un chef d'œuvre en péril ?}

La même préoccupation nourrit la réflexion des milieux d'affaires allemands sur l'isolement croissant dans lequel la cogestion place le système allemand de gouvernance face à l'émergence progressive d'un droit européen commun des sociétés. Après l'adoption de la directive sur les comités de groupe européens en 1994, la promulgation du statut de la société de droit européen en 2001, la jurisprudence de la Cour européenne de justice sur la liberté d'implantation des sociétés et l'adoption prochaine de la directive sur les fusions prévue à l'été 2005, le régime allemand de cogestion se trouve confronté à un processus d'ouverture et de mise en concurrence des dispositifs nationaux qui l'expose à un risque de marginalisation progressive.

La société de droit européen qui sert désormais de système de référence en cas de fusion ou de regroupement entre sociétés de droit national différent ne prévoit qu'une représentation salariée dans les organes de contrôle réduite au tiers des sièges. Pour les sociétés allemandes, ceci pose le problème du maintien ou de l'abandon du système paritaire et a justifié l'adoption d'un régime conservatoire. La transposition de ce dernier en droit allemand prévoit une négociation entre partenaires sociaux de la future société européenne, mais en cas d'échec, garantit l'adoption du régime de représentation le plus favorable, en l'occurrence le régime allemand. En cherchant ainsi à préserver sinon à étendre le droit national, le gouvernement allemand prend le risque de dissuader les partenaires potentiels et la création des sociétés de droit européen sur le sol allemand. L'alternative qui s'offrira alors aux sociétés allemandes qui souhaiteront s'orienter dans cette voie et bénéficier d'un régime moins contraignant sera alors de choisir une implantation dans un pays limitrophe, à l'instar de ce qui se fit lors de la création d'Aventis. L'adoption prochaine de la directive sur les fusions devrait encore accélérer le processus en affirmant plus clairement le principe selon lequel le droit des sociétés et le régime de gouvernance qui s'appliquera désormais en cas de fusion seront ceux du pays d'origine, c'est-à-dire en fait celui où la nouvelle société choisira d'implanter son siège. La porte se trouvera ainsi largement ouverte aux sociétés allemandes pour sortir de la cogestion paritaire en délocalisant leur siège, ce qui devrait précipiter l'érosion puis l'effritement du modèle.

Pour contrecarrer cet 'opting out' rampant, le prochain gouvernement allemand sera vraisemblablement conduit à sortir enfin de l'immobilisme et à s'engager sur la voie de la réforme avec le souci principal de rendre le modèle allemand de cogestion plus compatible avec les évolutions européennes en cours en matière de gouvernance, tout en maintenant un standard élevé de participation
Adoption d'un régime conservatoire outre-Rhin

Modernisation par dérégulation optionnelle? 
des salariés aux décisions. La modernisation du régime de cogestion devrait ouvrir la voie d'une dérégulation optionnelle en permettant aux entreprises de sortir du régime uniforme d'obligation légale et de choisir entre plusieurs modèles de gouvernance par la voie d'un accord contractuel passé entre la direction et le personnel. Dans le cas des régimes à conseils de surveillance, la représentation des salariés pourrait être ramenée au tiers des sièges moyennant la limitation du nombre des administrateurs, une revalorisation de leurs prérogatives de contrôle et des procédures de délibération plus efficaces. Dans le cas de figure d'un système unitaire, la représentation salariée pourrait être légalement garantie au sein d'un Conseil de consultation distinct du Board, mais doté de prérogatives précisément définies. Cette réforme ne marquerait pas obligatoirement la fin de la cogestion puisque celle-ci, là où elle existe, pourrait être pérennisée et aménagée par voie d'accord. Mais elle mettrait fin à son caractère exclusif en permettant que s'instaure en Allemagne un système pluraliste de gouvernement d'entreprise.

\section{La co-décision replacée au cœur du système : une hypothèse vraisemblable ...}

Cela ne devrait pas pour autant marquer la fin de l'exception allemande en matière de participation des salariés dans l'entreprise, ni conduire nécessairement à une régression sociale, car la co-décision telle qu'elle existe et telle qu'elle est pratiquée au niveau de l'établissement n'est pas fondamentalement remise en question par ces évolutions ; elle pourrait même s'en trouver confortée.

Influence croissante des Betriebsräte
Alors que, depuis les années 1990, la "grande cogestion " a de moins en moins permis aux mandataires salariés de peser effectivement sur les choix stratégiques, les Betriebsräte ont réussi à exercer une influence croissante et structurante dans la gestion de leurs conséquences sociales sur le terrain. Dotés de pouvoirs de co-décision très étendus, et jouissant d'un solide capital de confiance tant auprès des salariés que des directions d'entreprise par leur capacité éprouvée à concilier les intérêts des salariés et ceux de l'entreprise, ils se sont affirmés au fil des années, dans tous les secteurs, comme les acteurs d'un véritable co-management de l'entreprise. Et ce, dans les domaines les plus variés : ils se sont ainsi engagés dans la mise en œuvre de plans concertés de sauvegarde de l'emploi et de la compétitivité, ont assumé des politiques de flexibilité négociée du temps de travail et des rémunérations, se sont associés à la gestion des qualifications et à l'organisation du travail (Rehder, 2003), donnant même dans la dernière période leur aval à des délocalisations économiquement justifiées. Régulant ainsi en bon ordre les contraintes sociales internes et les effets de la globalisation, les BR sont parvenus, dans la sphère de l'activité productive, à contenir en partie la régression des acquis sociaux tout en contribuant à préserver la compétitivité des entreprises.

L'efficacité de la co-décision ne devrait donc pas directement souffrir du recul prévisible du pouvoir d'influence de la représentation salariée dans les organes statutaires de contrôle, dans la mesure où ce pouvoir était plus institutionnel que réellement opérationnel. Dans sa réalité, la co-surveillance relève davantage de l'observation stratégique et de l'intelligence économique des performances et marges de manoeuvre de l'entreprise que de l'exercice effectif d'un contre-pouvoir. En fin de compte, c'est à d'autres instances que revient la capacité de relayer et d'opérationnaliser ce pouvoir d'expertise, d'une part aux directions syndicales à travers la négociation des conventions de branches, d'autre part aux Betriebsräte à travers l'enjeu essentiel qu'est la gestion des conditions d'emploi et de travail dans l'activité quotidienne de l'entreprise. La réforme annoncée des conditions d'exercice du contrôle salarié ne signifie nullement sa disparition ni la fin de la transmission de l'information stratégique aux instances de représentation. Elle ne ferme pas non plus la porte à la revalorisation des prérogatives économiques du Betriebsrat, qui pourrait intervenir sinon par la 
voie légale, du moins par la voie contractuelle. Loin de devoir être perçue comme une régression, la refonte de la cogestion peut ouvrir de nouvelles voies à l'expérimentation et à l'exercice d'un réel co-management.

\section{...mais un pari incertain}

Restent néanmoins deux incertitudes majeures dont dépendra finalement l'avenir de la Mitbestimmung, prise au sens large, à l'issue de l'incontournable réforme de la cogestion paritaire. L'étendue et la portée réelle des pouvoirs de codétermination des travailleurs salariés dans l'entreprise ne dépendront plus, pour l'essentiel, que de l'existence et de la capacité d'action des Betriebsräte qui en resteront les vecteurs prépondérants. Or, en dépit de leur fonction primordiale, leur position dans le système allemand de régulation reste doublement fragile. Leur statut légal les protège certes du processus de désyndicalisation, mais il ne garantit pas pour autant leur existence effective, puisqu'ils sont très largement absents des petites et moyennes entreprises et de certains secteurs, notamment dans l'artisanat et les services et, parmi ces derniers, des nouvelles activités de service, en particulier celles de l'information et de la communication. De sorte que d'ores et déjà on assiste à une dualisation croissante de la régulation sociale d'entreprise entre des secteurs bien couverts et les secteurs délaissés. Cette tendance ne sera pas affectée par la réforme de la cogestion dans le conseil de surveillance des grandes sociétés. A moins qu'en contrepartie, le législateur veille à effectivement revaloriser le rôle des $\mathrm{Be}$ triebsräte en encourageant leur implantation, en améliorant leur fonctionnement et en renforçant leurs prérogatives.

L'autre enjeu, plus déterminant encore, est celui de leur capacité d'action et surtout de la logique selon laquelle celle-ci est susceptible d'évoluer en relation directe avec les mécanismes de la négociation conventionnelle. La crise du contrat unitaire de branche (Flächentarifvertrag) ouvre en effet un espace de négociation croissant au niveau de l'entreprise, lequel ne peut être juridiquement et fonctionnellement assuré que par les Betriebsräte, mais avec des moyens d'action limités (Lattard, 2005). La consolidation de leurs pouvoirs de codécision dans le contexte d'une refonte de la cogestion n'est cependant pas indéfiniment compatible avec l'extension de leurs pouvoirs de négociation découlant d'un dépérissement de la négociation de branche. Elle risque de se heurter non seulement à un délicat problème de surcharge, mais surtout à un conflit de logique : la négociation implique en effet une capacité revendicative et des moyens de pression conflictuelle qui sont difficilement compatibles avec la logique de coopération qu'implique la pratique du co-management.

LA GLOBALISATION DES MARCHES et l'intégration croissante de l'espace économique européen mettent à rude épreuve chacun des deux piliers fondamentaux du système allemand de relations sociales que sont la négociation sociale de branche et la gestion partenariale d'entreprise. Imposant à chacun de ces niveaux une différenciation croissante des enjeux et des modalités de régulation, elles ébranlent l'équilibre architectural d'ensemble du système et dérèglent sa mécanique de fonctionnement. La flexibilisation croissante de la négociation de branche et la reconfiguration du système de cogestion convergent ainsi vers une régulation d'entreprise décentralisée. Au risque de mener à terme vers l'émergence d'un système hybride et atomisé de représentation dans lequel s'affronteront des logiques contradictoires qui, face aux contraintes économiques et au recul de l'influence syndicale, s'affaibliront plus qu'elles ne se conforteront mutuellement.

Comment, dans un tel scénario, ne pas craindre que la pratique et la culture cogestionnaires auquel le mouvement social allemand a réussi à donner corps dans le contexte du renouveau démocratique et économique de l'après-guerre, 
puissent rapidement dépérir ? Que restera-t-il du grand projet social dont l'Allemagne démocratique a été porteuse pour l'Europe : celle d'une citoyenneté économique et sociale qui met la force créatrice du marché au service de l'épanouissement de l'individu?

\section{Indications bibliographiques :}

BDI, BDA, Mitbestimmung modernisieren. Bericht der Kommission Mitbestimmung, Berlin, décembre 2004

Bertelsmann Stiftung, Hans-Böckler Stiftung (eds), Mitbestimmung und neue Unternehmenskulturen, Gütersloh, 1999

Frick B. et al. (eds), Die wirtschaftlichen Folgen der Mitbestimmung, Francfort/Main, 1999

"Ist das deutsche Modell der Unternehmensmitbestimmung überholt ? » Diskussionsbeiträge, in Ifo-Schnelldienst 22/2004

JüRGENS U. et al., Corporate Governance und shareholder Value in Deutschland, Berlin, mars 2000

REHDER B., Betriebliche Bündnisse für Arbeit in Deutschland. Mitbestimmung und Tarifpolitik im Wandel, Francfort/Main, 2003

« Tarifliche Bündnisse und betriebliche Mitbestimmung », Aus Politik und Zeitgeschichte, Das Parlament, 55/22 (30-05-2005)

LATTARD, A., "Négociation collective : quel avenir pour la convention de branche ? " in : BOURGEOIS I. (dir), Le modèle social allemand en mutation, CIRAC, 2005 (à paraître en septembre 2005). 